\title{
Impacts of Porous Silica-Nanoencapsulated Pesticide Applied to Soil on Plant Growth and Soil Microbial Community
}

\author{
Vinicius Bueno ${ }^{1}$, Peiying Wang ${ }^{2}$, Orfeo Harrisson ${ }^{1}$, Stéphane Bayen $^{2}$ and Subhasis Ghoshal ${ }^{1 *}$ \\ ${ }^{I}$ Department of Civil Engineering, McGill University, Montreal, Quebec H3A OC3, Canada \\ ${ }^{2}$ Department of Food Science and Agricultural Chemistry, McGill University, Ste-Anne-de-Bellevue, \\ Quebec H9X 3V9, Canada
}

*Corresponding author, e-mail: subhasis.ghoshal@mcgill.ca, Phone: (1) 514-398-6867; Fax: (1) 514-398-7361 


\begin{abstract}
Porous silica nanocarriers have the potential to improve agricultural crop productivity. However, the impacts of nanoencapsulated pesticides on soil health and plant growth, and how they compare with conventional pesticide have not been systematically elucidated. In this study, we investigated how applying azoxystrobin encapsulated in porous hollow $\mathrm{SiO}_{2}$ nanocarriers to agricultural soil impacted the soil microbial community and plant development, using Solanum lycopersicum grown in the laboratory in soil microcosms. The data show that plant growth was heavily inhibited by the non-encapsulated pesticide treatment compared to that with encapsulated pesticide yielding 3.85-fold less plant biomass, while the soil microbial community experienced few to no changes regardless of the treatment. There was a 2.7-fold higher azoxystrobin uptake per unit dry plant biomass after 10 days of exposure for the non-encapsulated pesticide treatment when compared to that of nanoencapsulated pesticide, but only 1.5-fold increase in total uptake. After 20 days of exposure, however, the total uptake and uptake per unit of dry biomass were 3 -fold and 10-fold higher, respectively, for the nanopesticide treatment. The differences in uptake can be attributed to phytotoxicity caused by the high the bioavailability of the non-encapsulated pesticide. The nanocarrier promoted slow release of the pesticide over days, which prevented phytotoxicity, and allowed healthy plant growth.
\end{abstract}




\section{Environmental Significance}

Nanoencapsulated pesticides are viewed as promising agents for improving pesticide utilization rates in agriculture. While there has been significant recent focus on studying the feasibility and mechanisms of uptake of nanoparticles to plants, the bioavailability of organic pesticides to plants and its implications on plant health when encapsulated in inorganic nanocarriers is poorly understood. In this study, we reported higher uptake to azoxystrobin supplied in the nanoencapsulated form compared to those of the non-encapsulated form, following their application in soils. Furthermore, the silica nanocarrier in itself did not affect soil and plant health adversely, but more importantly, mitigated the toxic effect of high concentrations of pesticide towards plant growth. This indicates that nanoencapsulated pesticides can be more efficient in delivering organic pesticides and thus reduce the number of applications required, which will reduce pesticide and energy use. 


\section{Introduction}

There is growing interest in exploring the development and use of nanomaterials of various composition and forms for efficient delivery of pesticides, nutrients or other growth factors to crop plants, towards increasing crop yields and reducing agrochemical wastage and environmental contamination. ${ }^{1-5}$ Nanocarriers are a class of nanomaterials that can facilitate targeted delivery and controlled release of pesticides and fertilizers in plants. ${ }^{6}$ There is significant interest in using nanocarriers both for foliar or root applications. Although application of nanoformulations on leaves, rather than directly on soils, can better reduce fertilizer and pesticide wastage and avoid soil contamination, ${ }^{2-4}$ soil drenching with nanomaterial dispersions may be necessary to condition agricultural soils before the addition of seeds, to minimize pests and to provide optimal plant growth conditions. ${ }^{7-14}$ Nanocarriers may be provide advantages over conventional formulations by aiding the dispersion of hydrophobic chemicals, providing controlled release of the active ingredient, and prevent their abiotic or abiotic degradation in soil.

$\mathrm{SiO}_{2}$ nanoparticles (NPs) designed as porous structures have been recently suggested as promising nanocarrier for pesticides. $\mathrm{SiO}_{2}$ is an earth-abundant material, it is a potentially safe for use in agriculture. ${ }^{15-17}$ Enhanced uptake of silicon increased plant resistance to pathogens and abiotic stress. ${ }^{16,18-21}$ Furthermore, $\mathrm{SiO}_{2}$ NPs have been shown to enhance growth, protein content and photosynthesis in lupin, ${ }^{22}$ protect wheat against UV-B stress, ${ }^{23}$ chromium- and arsenic-induced oxidative stress, ${ }^{24,25}$ and improve its germination, ${ }^{26}$ root growth and chlorophyll content. ${ }^{27}$ Tian et al. ${ }^{28}$ reported that continuous application of $\mathrm{SiO}_{2} \mathrm{NPs}$ increased activity of enzymes associated with carbon and nitrogen cycling in soil. A few studies have shown that encapsulating pesticides within $\mathrm{SiO}_{2} \mathrm{NPs}$ could increase pesticide translocation and fungicidal activity, provide controlled and targeted delivery properties, and promote physical protection against premature degradation, but the application of pesticides encapsulated in $\mathrm{SiO}_{2}$ nanocarriers in soils have not been investigated. ${ }^{29-35} \mathrm{Xu}$ et al. ${ }^{35}$ reported that encapsulating pyraoxystrobin within a carbon nanotubemesoporous silica nanoparticle (MSN) composite and introducing it in growth medium, enhanced the upward translocation of the fungicide 3.5-fold in cucumber seedlings in hydroponic systems. Abdelrahman et al. ${ }^{29}$ developed a stimuli-responsive MSN to control the delivery of prochloraz to rice plants. Pectin was functionalized into the MSNs surface to cover the pores, acting as a gatekeeper compound, and when pectinase was added to the system, pectin was hydrolyzed, 
releasing the prochloraz. The authors reported enhanced pesticide translocation and fungal activity over a prolonged period when compared to conventional prochloraz formulations in aquatic microcosms.

Herein, we encapsulated azoxystrobin within porous hollow $\mathrm{SiO}_{2} \mathrm{NPs}$ (PHSNs) and examined its impacts on plant growth in soil systems as well the soil microbial population, and how they compare with non-encapsulated azoxystrobin applications following soil application. PHSNs are particularly promising nanocarriers because they allow for the high-density loading of molecules. Unlike MSNs, PHSNs have larger pore volume than MSN due to a void core, where one of the same or several different molecules can be encapsulated. Bueno and Ghoshal (2020) ${ }^{36}$ demonstrated that solutes can be transported across the porous shell of the PHSN. Azoxystrobin was selected because it is a commonly used fungicide for crop agriculture worldwide, is highly efficient, ${ }^{37}$ has broad spectrum coverage and is a systemic pesticide. Studies have reported phytotoxicity of azoxystrobin to apple and grape cultivars ${ }^{38-40}$ as well as to soil microbiota other than targeted pathogenic micoorganisms. ${ }^{41}$ Therefore, an ideal nanocarrier for azoxystrobin would mitigate its toxicity towards crops and vital soil microbiota by providing controlled release of the azoxystrobin. Assessing adverse effects of PHSN and the encapsulated azoxystrobin on soil microbiota is important to verify because they regulate nutrient cycling and carry out other important functions for plant growth. Major shifts in key soil microbial communities other than in the targeted pathogenic microorganisms can affect the soil health and the ability to cultivate crops.

In this work, we demonstrated the slower release of nanoencapsulated azoxystrobin from PHSN compared to the dissolution rate of the non-encapsulated azoxystrobin. We hypothesized that the nanoencapsulated form of azoxystrobin will have different uptake extents and distributions compared to the non-encapsulated form, and thus different impacts on plant health.

\section{Materials and Methods}

Materials. Tetraethyl orthosilicate (TEOS, reagent grade 98\%), ammonium hydroxide solution $\left(\mathrm{NH}_{4} \mathrm{OH}, 28.0-30.0 \% \mathrm{NH}_{3}\right.$ basis), hexadecyltrimethylammonium bromide (CTAB) and Pluronic P123 were purchased from Sigma-Aldrich. Deionized (DI) water ASTM type 1, Invitrogen UltraPure DNAse-, RNAse-free DI water, HPLC grade solvents (water, acetonitrile and methanol), formic acid, ammonium acetate, anhydrous magnesium sulphate $\left(\mathrm{MgSO}_{4}\right)$, dimethyl sulfoxide, 
and sodium acetate were purchased from Thermo Fisher Scientific. Primary and secondary amine (PSA) salt was purchased from Agilent. Anhydrous ethanol (100\%) was purchased from Commercial Alcohols (Canada). Solanum lycopersicum seeds were purchased from McKenzie Seeds (Canada). iTaq Universal SYBR Green Supermix was purchased from BioRad. Bovine serum albumin was purchased from New England BioLabs. Azoxystrobin $\left(\mathrm{C}_{22} \mathrm{H}_{17} \mathrm{~N}_{3} \mathrm{O}_{5}\right.$, CAS Registry Number 131860-33-8, log $\mathrm{K}_{\mathrm{ow}}$ 3.7) was provided by Vive Crop Protection Inc (Canada) in powder form. Deuterated internal standard ( $\mathrm{D}_{4}$-azoxystrobin) was purchased from Toronto Research Chemicals (Canada).

Soil was collected from an agricultural site at the Macdonald campus of McGill University. The soil was characterized by A\&L Canada Laboratories (Ontario, Canada) and the soil properties are shown in Table $\mathrm{S} 1$.

$\mathrm{SiO}_{2} \mathrm{NP}$ synthesis. Porous hollow $\mathrm{SiO}_{2} \mathrm{NPs}$ (PHSN) were synthesized as reported in our previous work. ${ }^{36}$ Briefly, in a round-bottom flask, $300 \mathrm{mg}$ of CTAB and $850 \mathrm{mg}$ of Pluronic were added to a mixture containing $75 \mathrm{~mL}$ of anhydrous ethanol and $125 \mathrm{~mL}$ of DI water. The surfactants selfassembled forming the hollow core for the $\mathrm{SiO}_{2} \mathrm{NP}$. Then, $15 \mathrm{~mL}$ of $\mathrm{NH}_{4} \mathrm{OH}$ is added to alkalinize the medium and accelerate the hydrolysis of the silica precursor, TEOS, which was added dropwise at a rate of $0.75 \mathrm{~mL} / \mathrm{min}$ for $13.33 \mathrm{~min}$. The reaction was allowed to proceed for 5 hours under vigorous magnetic stirring. The suspension was dried overnight under $80{ }^{\circ} \mathrm{C}$ and calcined under $550^{\circ} \mathrm{C}$ for $5 \mathrm{~h}$ to remove any trace of surfactants and ammonia by-products. Detailed characterization data for PHSN has been reported in our prior studies and the primary particle diameter was determined by transmission electron microscopy to be $253 \mathrm{~nm}$ in diameter, ${ }^{36}$ with a porous shell of thickness ranging from 22 to $38 \mathrm{~nm} .{ }^{36} \mathrm{BET}$ analysis indicated pores in the order of $1.5 \mathrm{~nm},{ }^{36}$ and a specific surface area of $287 \mathrm{~m}^{2} \mathrm{~g}^{-1} \cdot{ }^{36}$ Electrophoretic mobility measurements provided ae zeta potential of $-29.2 \pm 1.1 \mathrm{mV}$ at $\mathrm{pH} 6.5$ and ionic strength $1 \mathrm{mM}(\mathrm{NaCl}){ }^{42}$

Azoxystrobin loading and release rates. $0.1 \mathrm{mg} / \mathrm{mL}$ of azoxystrobin was solubilized in a mixture containing DI water (80\%) and methanol (20\%). Without methanol, most of the azoxystrobin added at this concentration would remain in its solid phase due to its low aqueous solubility (6 $\mu \mathrm{g} / \mathrm{mL}$ ). Furthermore, when solubilized, the solute (azoxystrobin) is able to infiltrate the pores and reach the hollow core of the PHSN. ${ }^{36}$ These experiments were performed in Nalgene Teflon tubes to avoid azoxystrobin sorption onto the container's walls. This ratio was determined by measuring 
the dissolved azoxystrobin in mixtures of varying water-methanol ratios, to identify low methanol doses that provide sufficient solubilization of azoxystrobin. Then, PHSN were added to the system at a concentration of $0.67 \mathrm{mg} / \mathrm{mL}$. The tubes were stirred at $200 \mathrm{rpm}$. Solution aliquots were collected at specific time intervals, diluted in acetonitrile, and analyzed using an Agilent 1260 Infinity II HPLC system equipped with UV detector (at $255 \mathrm{~nm}$ characteristic wavelength), using acetonitrile and water $(80: 20 \mathrm{v} / \mathrm{v})$ as the mobile phase. At the $8^{\text {th }}$ day, the PHSN were separated from the solution through centrifugation under $5500 \mathrm{rpm}$ for $15 \mathrm{~min}$. The final azoxystrobin concentration in the solution and loaded in the PHSN were quantified in the HPLC-UV to account for the mass balance. The loaded azoxystrobin was extracted from the PHSN through successive acetonitrile extractions until no further azoxystrobin could be extracted.

The release time profile of the nanoencapsulated azoxystrobin was obtained by dispersing the azoxystrobin-loaded PHSN in a mixture of DI water-methanol (80:20 v/v). Due to azoxystrobin's low aqueous solubility as previously discussed, the release profile would be limited by the dissolution rate. The addition of methanol eliminates the solubility limitation and allows the investigation of the release profile of the pesticide in the PHSN hollow core and porous shell and as well from the solid phase non-encapsulated azoxystrobin. Aliquots were collected at different time intervals and analyzed in HPLC-UV. The dissolution profile for pure azoxystrobin was obtained to compare the release profiles of the pure compound and the loaded compound.

Plant growth conditions and soil amendment. The Solanum lycopersicum seeds were sterilized in a $1.5 \%$ sodium hypochlorite solution for $15 \mathrm{~min}$ and then rinsed thoroughly with DI water. The seeds were covered in slightly wet tissue paper placed inside Petri dishes and allowed to germinate for 10 days in total darkness. Seedlings at the same growth stage were carefully picked and transplanted to soil. Each pot contained approximately $61.6 \mathrm{~g}$ of dry soil and the humidity was kept at $70 \%$ of the water holding capacity at 1/3 bar. Four different treatments were analyzed to assess the impact of nanoencapsulated pesticides (i) Azo@PHSN: For the encapsulated pesticide, the soil was homogeneously amended with a suspension containing $5 \mathrm{mg}$ of $\mathrm{SiO}_{2} \mathrm{NPs}$ and 0.75 mg of azoxystrobin in $7.5 \mathrm{~mL}$ of an 80:20 water-methanol mixture. (ii) Azo: For the nonencapsulated pesticide formulation, the soil was amended with $0.75 \mathrm{mg}$ of azoxystrobin in $7.5 \mathrm{~mL}$ of an 80:20 water-methanol mixture (v/v). (iii) PHSN: A control with only $\mathrm{SiO}_{2} \mathrm{NPs}$ amendment was prepared by suspending $5 \mathrm{mg}$ of $\mathrm{SiO}_{2} \mathrm{NPs}$ in a $7.5 \mathrm{~mL} \mathrm{80:20}$ water-methanol mixture. (iv) 
Lastly, a control amendment was prepared by homogenizing a 7.5 mL 80:20 water-methanol mixture in soil to assess the impact of the water-methanol mixture would have itself in the plant growth and soil microbial community. It is important to note that the soil was amended with more than the recommended amount of azoxystrobin established by the EPA, ${ }^{43}$ Health Canada ${ }^{44}$ and commercial suppliers. This was done to ensure observable effects of the pesticide and quantitatively assess if the $\mathrm{SiO}_{2}$ nanocarrier was able to mitigate the pesticide toxicity/inhibitory effects. The pots were placed randomly and equidistant in an incubator with controlled conditions for 20 days: relative humidity at $70 \pm 5 \%$, a light/dark cycle of 16:8 h, and constant temperature of $21 \pm 1^{\circ} \mathrm{C}$.

Plant health data. The plants $(N=3)$ were harvested at days 10 and 20. The plant health was assessed by quantifying five observable characteristics and traits of the plants, including dry biomass (mg), root length $(\mathrm{cm})$, shoot length $(\mathrm{cm})$, number of leaves, and length of the longest leaf $(\mathrm{cm})$. Statistical analysis (ANOVA one-way, $\mathrm{p}<0.05$ ) was performed to identify significant statistical differences among the phenotypic traits assessed.

Azoxystrobin uptake measurements in plants. The plants were thoroughly rinsed with DI water and dried overnight at $110^{\circ} \mathrm{C}$. Then the dried biomass was homogenized followed by pesticide extraction using a modified version of the QuEChERS (quick, easy, cheap, effective, rugged, and safe) method. ${ }^{45}$ In summary, a mixture containing $4 \mathrm{~mL}$ of acetonitrile in $1 \%$ acetic acid, $0.8 \mathrm{~g}$ of $\mathrm{MgSO}_{4}$, and $0.2 \mathrm{~g}$ of sodium acetate were added to each $0.2 \mathrm{~g}$ of homogenized biomass $(N=3)$ for each treatment. The mixture was vortexed for $15 \mathrm{~min}$, followed by centrifugation at $2240 \times \mathrm{g}$ for 5 min under $20^{\circ} \mathrm{C}$ and recovery of the supernatant. Then, $1 \mathrm{~mL}$ of the supernatant was transferred to centrifuge tubes containing $50 \mathrm{mg}$ of PSA and $150 \mathrm{mg}$ of $\mathrm{MgSO}_{4}$, which were further vortexed for $1 \mathrm{~min}$, followed by another step of centrifugation at $2240 \times \mathrm{g}$ for $5 \mathrm{~min}$ under $20^{\circ} \mathrm{C}$. Finally, the supernatant was passed through a $0.22 \mu \mathrm{m}$ polytetrafluoroethylene (PTFE) filter before proceeding to the measurements. Azoxystrobin quantification from the extract solution was performed in an Agilent 1290 Infinity II liquid chromatograph (LC) coupled to a quadrupole timeof-flight mass spectrometer (QToF-MS) (Agilent) operating in positive electrospray ionization mode. The method validation for the recovery rates in homogenized plant biomass was assessed in the Table S2. Azoxystrobin was extracted and measured from soil based on a protocol described previously. ${ }^{46} 1 \mathrm{~g}$ of soil dried in room temperature was shook with $2 \mathrm{~mL}$ of acetonitrile for $1 \mathrm{~h}$ 
under $20 \mathrm{rpm}$ on a vertical shaker, followed by centrifugation $(1882 \times \mathrm{g}$ for $5 \mathrm{~min})$ and filtration (0.22 $\mu \mathrm{m}$ PTFE filter) steps before LC analysis.

DNA extraction and sequencing. Bulk soil $(N=3)$ and soil loosely attached to roots $(N=3)$ were collected at days 10 and 20. The genomic DNA was extracted from approximately $250 \mathrm{mg}$ of dry soil using a DNeasy PowerSoil Pro kit (Qiagen). The extracted DNA concentration was quantified using the PicoGreen method ${ }^{47,48}$ (Invitrogen Quant-iT PicoGreen dsDNA Assay Kit, Thermo Fisher) to make sure it was within the range required by Genome Québec (Canada). The V4 region of the 16S rRNA gene in archaea and bacteria was amplified using the primers 515F (5'GTGCCAGCMGCCGCGGTAA-3') and 806R (5'-GGACTACHVGGGTWTCTAAT-3'). The ITS1 region of the ITS rRNA gene in fungi was amplified using the primers ITS1F (5'CTTGGTCATTTAGAGGAAGTAA-3') and 58A2R (5'-CTGCGTTCTTCATCGAT-3'). The amplified sequences were sequenced on Ilumina MiSeq using the PE250 protocol.

Sequencing data analysis. The sequence reads were processed using QIIME2 (version 2019.4) pipeline. ${ }^{49}$ The processing included (i) pairing forward and reverse sequence reads, (ii) demultiplexing sequences by linking the barcode information with the corresponding samples, (iii) denoising the amplicon sequence data with DADA2 pipeline ${ }^{50}$ and truncating at position 240 where quality started to drop significantly. Taxonomic ranks were assigned to the 16S rRNA processed sequences using Naïve Bayes Taxonomic Classifier ${ }^{51}$ trained with the Greengenes database. ${ }^{52}$ Taxonomic ranks were assigned to the ITS rRNA processed sequences fitting a classifier for the UNITE database ${ }^{53}$ and training it with a fungal ITS mock community published in Taylor et al. ${ }^{54}$ Alpha $(\alpha)$ diversity metrics (Faith phylogenetic diversity (PD) ${ }^{55}$ and Pielou's evenness indices ${ }^{56}$ ), and beta $(\beta)$ diversity metrics (weighted UniFrac ${ }^{57}$ ) for the Principal Coordinate Analysis (PCoA) were performed through the q2-diversity pipeline.

Quantitative Polymerase Chain Reaction (qPCR). To determine bacterial and fungal population size changes in each treatment, relative qPCR quantifications of the $16 \mathrm{~S}$ rRNA and 18S rRNA genes for bacteria and fungi, respectively, were undertaken. Universal primers, Ba519F and Ba907R, for bacteria community and, Fung5F and FF390R, for fungi community, were used as previously reported by Lueders et al. ${ }^{58,59}$ A standard curve was established using 10-fold dilutions of soil genomic DNA (gDNA) $\left(10^{0}-10^{-4}\right)$. Each $20 \mu \mathrm{L}$ reaction contained $10 \mu \mathrm{L}$ of iTaq Universal SYBR Green Supermix, $500 \mathrm{nM}$ of each primer, $0.2 \mu \mathrm{g} / \mu \mathrm{L}$ bovine serum albumin, $5 \%$ dimethyl 
sulfoxide, and $1 \mu \mathrm{L}$ of the serial dilutions of the standard soil gDNA, or 100-fold dilution of unknown gDNA for bacteria and 20-fold dilutions for fungi. The reaction was run on a CFX connect real-time PCR detection system (BioRad). The thermal cycling parameters can be found in Supporting Information. The specificity of each reaction was verified after each run through a melting curve analysis between $65^{\circ} \mathrm{C}$ and $95^{\circ} \mathrm{C}$. The efficiency of the reaction was calculated to be above $90 \%$ for both, and the purity of reagents was verified by running no template controls.

\section{Results and Discussion}

PHSN encapsulation provides controlled release properties. The decrease in the concentration of azoxystrobin over time in the solution phase outside the particles indicates the loading of the fungicide within the PHSN (Figure 1a). These data suggest that adsorption of azoxystrobin on the PHSN pore walls is the dominant process for loading of the PHSN, because only the seepage of the solution phase into the pores and hollow core of the dry PHSN would not have lowered azoxystrobin concentration in the exterior solution phase. We compared the loading rates of PHSN with solid $\mathrm{SiO}_{2}$ NPs with similar sizes $(\sim 200-250 \mathrm{~nm})$ as shown in Figure $\mathrm{S} 1$, which suggests that surface area plays a dominant role in the loading capacity of the nanocarrier. While SSNs (specific surface area: $29 \mathrm{~m}^{2} \mathrm{~g}^{-1}$ ) did not sorb a significant amount of the pesticide, PHSN (specific surface area: $287 \mathrm{~m}^{2} \mathrm{~g}^{-1}$ ) were loaded with azoxystrobin 14-fold higher than that of SSN after 8 days of contact in solution. The mass balance of azoxystrobin at the end of the experiment in day 14 was satisfactory, with $66.9 \pm 1.4 \%$ of the initial azoxystrobin mass was extracted from the PHSNs, and $42.2 \pm 3.4 \%$ of remained in solution, accounting for approximately of $107.8 \%$ of the initial azoxystrobin in the system. Most of the loading of azoxystrobin within PHSNs took place in the first 2 days, and the solution phase azoxystrobin concentrations plateaued after day 3 , as shown in the loading profile in Figure 1a.

Following the loading, the PHSN suspension was centrifuged to separate the PHSNs from the solution, and the pellets were introduced in a release medium similar to the loading medium (20\% v/v methanol solution), to compare the release profile of nanoencapsulated azoxystrobin and the non-encapsulated fungicide. Figure $1 \mathrm{~b}$ shows that non-encapsulated and nanoencapsulated azoxystrobin had considerably distinct release profiles in the release medium. All the non- 
encapsulated azoxystrobin was dissolved after few hours, whereas the nanoencapsulated azoxystrobin followed a controlled, prolonged release that extended over days. In fact, only 43.5 $\pm 5.8 \%$ of azoxystrobin was released after 240 hours. Due to the high loading of azoxystrobin, excessive dosing of PHSN is not required, making the process cost-effective.

Azoxystrobin uptake profiles differed significantly among the treatments. Although the same amount of azoxystrobin $(0.75 \mathrm{mg}$ ) was added in the Azo and Azo@PHSN treatments, the azoxystrobin uptake varied significantly among them. Figure $2 \mathrm{a}$ shows that the concentration of azoxystrobin per unit mass of the plant dry weight (dw) for the non-encapsulated treatment (Azo) at $12.77 \pm 0.68 \mathrm{mg} / \mathrm{kg} \mathrm{dw}$ at day 10 was more than double the nanoencapsulated treatment (Azo@PHSN). In terms of azoxystrobin mass uptake (Figure 2b), there was no significant difference in the uptake between Azo and Azo@PHSN treatments on day 10, and thus the changes in concentration were primarily related to the change in plant biomass (Figures $2 \mathrm{c}$ and $3 \mathrm{a}$ ). At day 20, the azoxystrobin concentration in the plants with the Azo treatment decreased to $0.89 \pm 0.11$ $\mathrm{mg} / \mathrm{kg} \mathrm{dw}$, which is partly attributable to an increase in the biomass, and partly due to a decrease in the mass of azoxystrobin present in the plants (Figures $2 \mathrm{~d}$ and $3 \mathrm{a}$ ). The latter suggests that the plants were able to metabolize and/or excrete the fungicide through guttation droplets from leaves and through roots exudates. ${ }^{60}$ Evidence of azoxystrobin metabolism inside the plants with both Azo and Azo@PHSN was confirmed by identifying peaks for suspected azoxystrobin metabolites by LC-MS. This indicates that the nanoencapsulated azoxystrobin was bioavailable to the plant tissues. The list of suspected metabolites found in plants for different treatments and time points is presented in Table S3. On the other hand, the concentration of azoxystrobin in plants for the nanoencapsulated treatment (Azo@PHSN) did not differ by much from day 10 to day 20, decreasing from $4.72 \pm 0.14$ to $2.65 \pm 0.13 \mathrm{mg} / \mathrm{kg} \mathrm{dw}$, respectively. However, for that treatment, azoxystrobin mass uptake increased 3.4-fold between days 10 and 20 and was accompanied by significant increases in biomass growth (Figures $2 \mathrm{c}, 2 \mathrm{~d}$ and $3 \mathrm{a}$ ), which resulted in relatively constant azoxystrobin concentrations in the plant, suggesting the sustained uptake of pesticide over time during an active growth period.

The azoxystrobin plant uptake efficiency (ratio of pesticide mass uptake to dosed) was $1 \%$ for the Azo treatment) to 5\% for the Azo@PHSN treatment over the 20-day experiment, indicating that nanoencapsulation leads to more efficient pesticide uptake. Xu et al. ${ }^{35}$ reported comparable uptake 
efficiencies for another kind of strobilurin pesticide, pyraoxystrobin. The non-encapsulated form yielded 1.5\% while the MSN-encapsulated form yielded 3.5\% of uptake after 10 days. Plant-free soil microcosms dosed with the azoxystrobin showed that the concentration of azoxystrobin in soil did not decrease after 20 days, indicating that biodegradation is not playing a role in the differences of uptake in the nanoencapsulated and non-encapsulated systems in this work. Si uptake in plants was not monitored because of the limitations to identify what fraction constitutes the Si from the engineered NPs and that from naturally occurring $\mathrm{SiO}_{2}$ in the environment. Moreover, the amount of $\mathrm{Si}$ added to the system is significantly below the background level of $\mathrm{Si}$ in soil, and plants can accumulate $\mathrm{Si}$ in the form of $\mathrm{SiO}_{2}$ that could potentially lead to the biosynthesis of $\mathrm{SiO}_{2} \mathrm{NPs}^{6}{ }^{61}$

\section{Azoxystrobin when loaded in $\mathrm{SiO}_{2}$ porous nanocarrier minimized its inhibitory effects on}

plants. The plant health was quantified by monitoring five measurable indicators in the plants harvested in days 10 (Figure 2b) and 20 (Figure 2c). These observable traits included the plant biomass (dry wt), the number of leaves, and the length of the roots, shoot and longest leaf. The quantitative measure for each trait, separated by treatments, is shown in Figure 3. ANOVA oneway analysis was performed to identify significant statistical differences among the treatments and the error bars represent the standard deviation for each treatment $(N=3)$.

The dry biomass at day 10 varied little among control, nanoparticle only (PHSN), and encapsulated azoxystrobin (Azo@PHSN) treatments. However, the treatment with non-encapsulated pesticide (Azo) yielded 4.4-fold, 5.2-fold and 3.8-fold less biomass than control, PHSN and Azo@PHSN treatments, respectively. It is evident that at the concentration applied, azoxystrobin caused inhibitory effects on the plant growth. However, the Azo@PHSN treatment resulted in biomass yield somewhat in between the PHSN and Azo treatments, with no statistical difference compared to control $(\mathrm{p}>0.05)$. The same trends in the pesticide concentration and biomass yield were observed at day 20. This suggests that the non-encapsulated azoxystrobin is somehow inhibiting plant growth and the PHSN might be limiting the negative effects of high levels of pesticide exposure to a certain extent by releasing it over time, thus mitigating its toxicity. Azoxystrobin has been shown to cause toxicity towards some varieties of cultivars. ${ }^{38-40,62,63}$

Similarly, the length of the roots at day 10 and 20 was similar among the control, PHSN and Azo@PHSN treatments, leading to no significant statistical difference among the three treatments 
$(\mathrm{p}>0.05)$. The Azo treatment yielded the smallest root length. The Azo treatment led again to the least root development.

There was no significant difference in the shoot length between control and PHSN treatments at days 10 and 20 of harvesting. The shoot development was somewhat impacted at day 10 for both azoxystrobin treatments (Azo and Azo@PHSN), but at day 20 the shoot development was evident for the Azo@PHSN. The Azo treatment continued to inhibit the plant development in a greater extent than the other treatments.

To some extent, the results for the other two observable indicators (number of leaves and length of the longest leaf) follow similar trends as the previous three observable traits: (i) Azo@PHSN overall showed little difference to control and PHSN, except for dry mass and length of the longest leaf, for which the treatment yielded lower values. (ii) Azo treatment always yielded the indicators with the lowest values, which suggests that azoxystrobin caused inhibitory effects on the plant development. (iii) PHSN treatment in all cases yielded similar results when compared to control, with the exception to the number of leaves indicator where PHSN treatment yielded significantly more leaves than control $(\mathrm{p}<0.05)$.

Overall, the growth of Solanum lycopersicum was negatively impacted by the pesticide-dosed treatments. While the treatment with non-encapsulated azoxystrobin yielded the lowest amount of biomass and highest growth inhibition, the treatment with nanoencapsulated azoxystrobin was placed somewhat in between the treatment with azoxystrobin and controls with respect to those parameters. The treatment with the nanoencapsulated azoxystrobin yielded less growth inhibition because the release profile of azoxystrobin in a porous nanocarrier was controlled and slowly released the AI for days, while the treatment with non-encapsulated azoxystrobin resulted in an immediate dissolution. The uptake per unit of dry biomass profiles in Figure 3 suggest that azoxystrobin was rapidly available in the Azo treatment and decreasing rapidly from then on, while the nanoencapsulated treatment released the pesticide in a controlled manner over time, which helped minimize the phytotoxic effects observed with the Azo treatment.

Nanopesticides had limited impact on the soil microbial community structures. Between 95 to $99 \%$ of the pesticide applied remained in the soil after nanoencapsulated and non-encapsulated pesticide treatments over the 20-day experiment. Therefore, the effect of the remaining azoxystrobin on the soil microbiota should also be investigated to grasp the full impact of applying 
nanoencapsulated pesticides and how that compare with non-encapsulated pesticide or no pesticide at all.

There are conflicting results about the azoxystrobin impacts on the microbial community in the literature. While some studies reported azoxystrobin reduced microbial diversity by inhibiting the growth of a number of bacteria and fungi in soil, ${ }^{64-66}$ particularly its target pathogens, Deuteromycetes, Ascomycetes, Basidiomycetes and Oomycetes, ${ }^{37}$ others have suggested that the fungicide had little to no significant effect on soil microbial communities. ${ }^{67-69}$ Such contrasting conclusions are likely attributed to differences in the soil properties, fungicide doses and application methods used in each study.

The level of richness and diversity in the soil microbiota is a key indicator to assess the health of the soil because the microbial communities work symbiotically to maintain its fertility, productivity and sustainability. ${ }^{70}$ A diverse consortium of soil bacteria drives nitrogen and carbon cycling ensuring proper nutrient distribution, ${ }^{71}$ suppression of (a)biotic stresses due to pathogens, ${ }^{72}$ drought, high salinity and metabolization of pesticides. ${ }^{73}$ Fungi are normally responsible for nutrient translocation and metabolization of biomolecules, enhancing nutrient availability to other microorganisms. ${ }^{74}$ Therefore, any disruption or deregulation in the soil microbiota may have serious consequences to the whole ecosystem. Figure 4 and Figure 5 show the taxonomic abundance of fungi at class level and bacteria and archaea at phylum level, respectively.

With few exceptions, there was no significant effect on the fungi community coming from any of the treatments in terms of relative abundance, for both bulk soil and the soil loosely attached to the roots. The soils treated with azoxystrobin saw a significant increase $(\mathrm{p}<0.05)$ in Saccharomycetes of 44-fold at day 10 and of 15-fold at day 20 when compared to the soils without pesticide dosing (control and PHSN treatments). The increase of 5 to $6 \%$ in relative abundance of Saccharomycetes was accompanied by an evenly decrease of all the other classes between 1 and $2 \%$. Saccharomycetes play an important role in mineralization processes and nutrient cycling in soil. ${ }^{75}$ In both cases, it is noticeable that when differences occurred, they tended to be more significant at day 10 than at day 20. This suggests that the soil microbiota acclimated to the pesticides over time. Nonetheless, the small differences in taxonomic relative abundance suggest that microbial community changes are not the driver for the differences in plant growth reported above. 
Similarly, with few exceptions, there was no significant effect on the bacterial community composition for any of the treatments, for both bulk soil and the soil loosely attached to the roots. The soils treated with azoxystrobin (Azo and Azo@PHSN treatments) experienced higher relative abundance of Proteobacteria on day $10(+6.7 \%)$, but relatively no difference at day 20 when compared to the soils without pesticide dosing (control and PHSN treatments). Among key functions in the soil, Proteobacteria are generally associated with cycling of nitrogen, sulphur and carbon. ${ }^{76}$ On the other hand, the soils treated with azoxystrobin (both as Azo and Azo@PHSN) experienced lower relative abundance of Acidobacteria at days $10(-6.9 \%)$ and $20(-4.0 \%)$. Acidobacteria is very sensitive to change in soil physicochemical properties, ${ }^{77}$ thus the addition of pesticides likely negatively disrupted this phylum.

The PCoA for the bacterial and archaea communities (Figure 6a) revealed that there are two parameters affecting the $\beta$-diversity (the measurement of the change in diversity of species from one system to another) of these communities: (1) time, and to a smaller extent (2) different treatments. There are two distinct clusters based on time periods, one that includes the triplicates from day 0 , most treatments from day 20 and azoxystrobin-free treatments from day 10, located mostly in the border between $Q 1$ and $Q 4$, and the other cluster includes the treatments involving azoxystrobin at day 10 which are primarily in $Q 2$ and $Q 3$. Furthermore, the pesticide treatments at day 10 are further away from the initial soil stage (at day 0) than the same treatments at day 20. Therefore, the pesticide-based treatments had a more significant impact on the $\beta$-diversity of community compared to other treatments. This effect, however, was more significant in the first 10 days, and the microbial community in the soils tended to bounce back to their initial stage as the day progressed until day 20. Similarly, the PCoA for the fungi communities (Figure 6b) revealed that pesticide-dosed treatments and time also influenced the $\beta$-diversity of these communities. Although the different treatment triplicates are more spread out in the fungi communities PCoA, the pesticide-dosed treatments located mostly in $Q 2$ and $Q 3$ are still the furthest away from the initial stage triplicates at day 0 in $Q 4$, and as time progressed to day 20 , the triplicates tended to bounce back to the initial stage. This is another instance where the data suggests that the soil microbiota acclimated to azoxystrobin. While relative abundance did not change considerably among samples (Figures 4 and 5), $\beta$-diversity shifts are more evident (Figure 6), suggesting that although the pesticide treatments (Azo and Azo@PHSN) did not affect the 
relative abundance, they seemed to have an effect in the overall richness and evenness of the samples when compared to the ones treated without pesticides (control and PHSN).

Furthermore, $\alpha$-diversity (the measurement of the change in diversity within one sample) analyses were performed to investigate whether there are statistically significant differences among treatments. Overall, the $\alpha$-diversity analysis indicated that there were a few differences among treatments, mostly associated with treatments dosed with pesticides (Figure S2). Lo ${ }^{78}$ reported that soils with high organic matter content enhance sorption of hydrophobic agrochemicals, such as azoxystrobin, making them unavailable to microorganisms. Thus, hydrophobic pesticides may have minimal effects on microbial community in soils with abundant organic matter. These results suggest that the toxic effect of azoxystrobin may be a direct effect on the plant, rather than an effect on the soil microbiota which then would affect the plant growth.

The qPCR data for bacterial and archaea communities (Figure S3) and fungi communities (Figure S4) confirmed the trends observed with the relative abundance and diversity analyses. The bacterial and archaea community population size changes (Figure S3) do not vary significantly compared with control at day 0 or the control systems at day 10 and 20 to an extent that would explain the abrupt inhibition in plant growth that was observed during Azo treatments. The fungi community population size changes (Figure S4) showed that Azo@PHSN was the only instance where the community numbers decreased when compared to day 0 , particularly at day 10 for bulk soil and soil loosely attached to the roots. The continuous supply of the pesticide over a longer period of time promoted by the encapsulated formulation likely was responsible for decreasing the overall fungi community numbers, while a one-dose non-encapsulated pesticide formulation was not able to maintain the biocidal effects for the same period of time.

\section{Conclusion}

In this study, the pesticide encapsulation promoted the slow, controlled release of the AI over days, whereas the non-encapsulated formulation of pesticide experienced a rapid dissolution within hours. This controlled release mitigated the impacts of the pesticide in the plant health. Plants in soils which were treated with non-encapsulated azoxystrobin yielded less biomass than those treated with nanoencapsulated azoxystrobin. In terms of soil microbiota, all treatments led to 
relatively minor changes in the soil microbial communities. It is unlikely that these shifts influenced the plant growth, because key bacterial and fungi communities responsible for soil health, were not heavily impacted.

Overall, the use of nanocarriers to encapsulate and transport AIs did not seem to negatively impact the plant health and soil microbial community, rather it helped mitigate the phytotoxicity by slowly releasing the pesticide over time. The nano-encapsulated azoxystrobin was bioavailable in the plants as evidenced by generation of its metabolic products. The relatively small mass of PHSN added in soil (5 mg per $61.6 \mathrm{~g}$ soil) led to a five-fold increase in azoxystrobin uptake, with almost no phytotoxicity. In our prior study, we also found that the PHSN have limited mobility in subsurface granular media, due to their high surface roughness ${ }^{42}$. These are particularly promising results for pesticide application of silica nanocarriers and imply that fewer pesticide applications may be necessary with the use of nanocarriers. However, these results need to be verified with different soil types and crops.

\section{CRediT authorship contribution statement}

Vinicius Bueno: Conceptualization, Methodology, Investigation, Writing - original draft, Visualization. Peiying Wang: Conceptualization, Methodology, Investigation, Writing - review \& editing. Orfeo Harrisson: Conceptualization, Methodology, Investigation, Writing - review \& editing. Stéphane Bayen: Conceptualization, Investigation, Writing - review \& editing, Supervision, Funding acquisition. Subhasis Ghoshal: Conceptualization, Investigation, Writing review \& editing, Supervision, Funding acquisition.

\section{Conflicts of Interest}

There are no conflicts of interest. 


\section{Acknowledgments}

The research was funded by the Natural Sciences and Engineering Research Council of Canada (grant nos. RGPIN-2016-05022, STPGP 506450-17) and the Fonds de Recherche du Québec Nature et Technologies (grant no. 286120). Funding from the Canada Foundation for Innovation/John R. Evans Leaders Fund grant (Project \#35318) to S.B is acknowledged. V.B. was supported in part by a McGill Engineering Doctoral Award. 


\section{Figures}

\section{(a)}
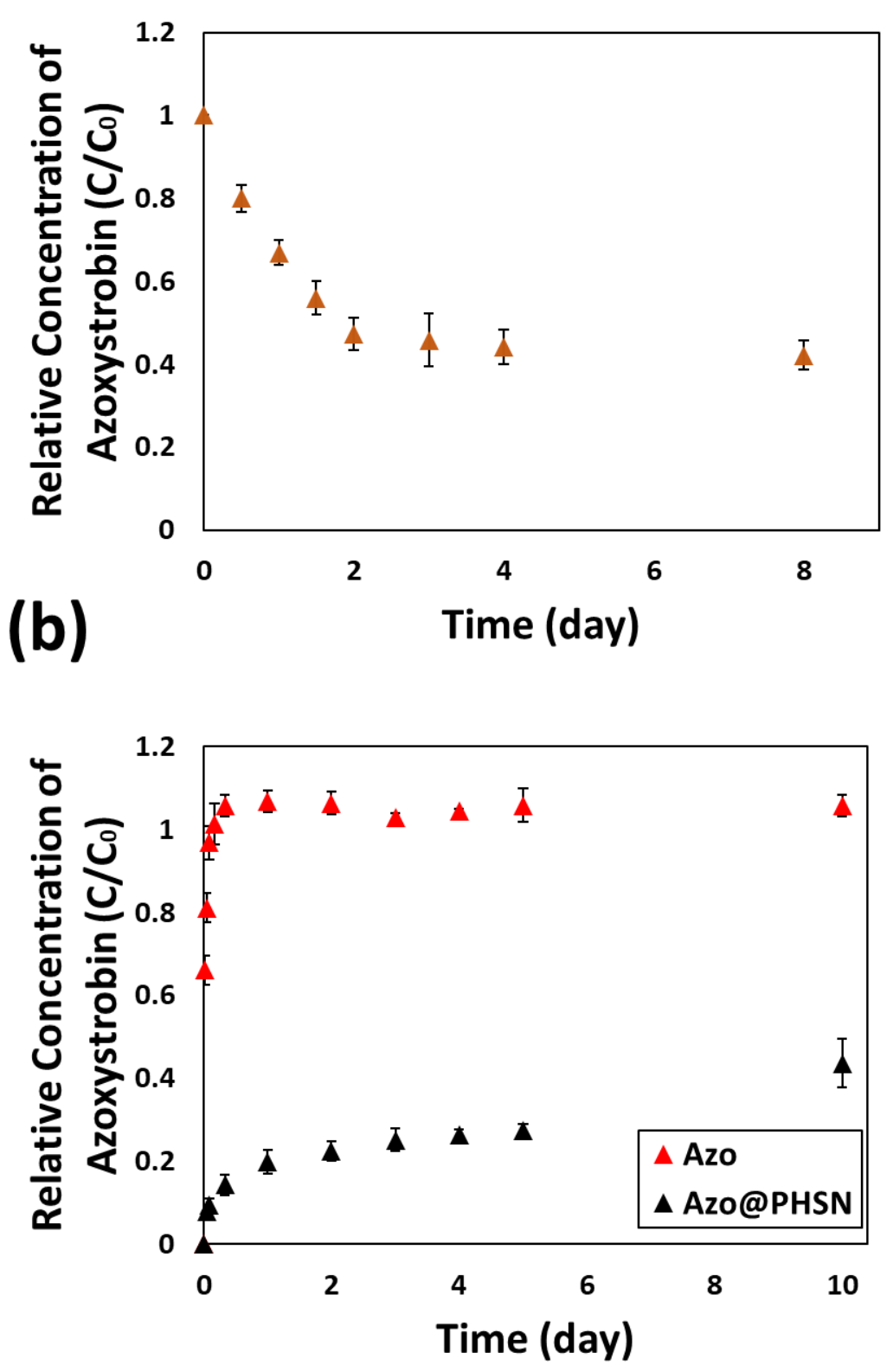

Figure 1. (a) Relative concentration $\left(\mathrm{C} / \mathrm{C}_{0}\right)$ of azoxystrobin in solution ( $20 \% \mathrm{v} / \mathrm{v}$ methanol) phase over time and (b) Concentration of azoxystrobin over time in solution ( $20 \% \mathrm{v} / \mathrm{v}$ methanol) released from pure (Azo) and encapsulated azoxystrobin (Azo@PHSN). 
(A)

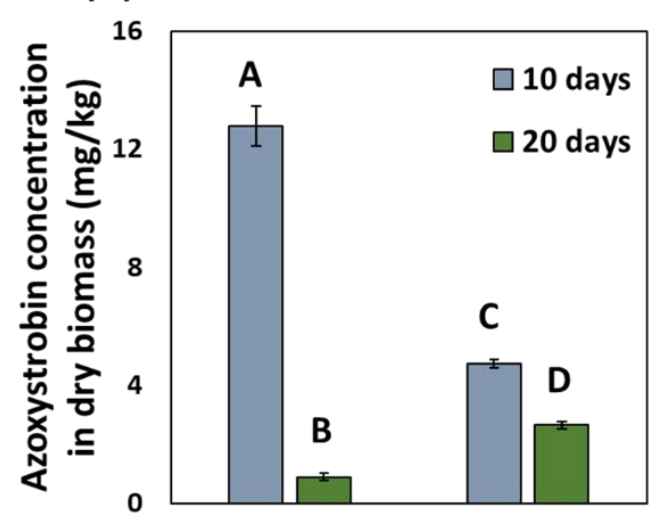

(B)

Azo

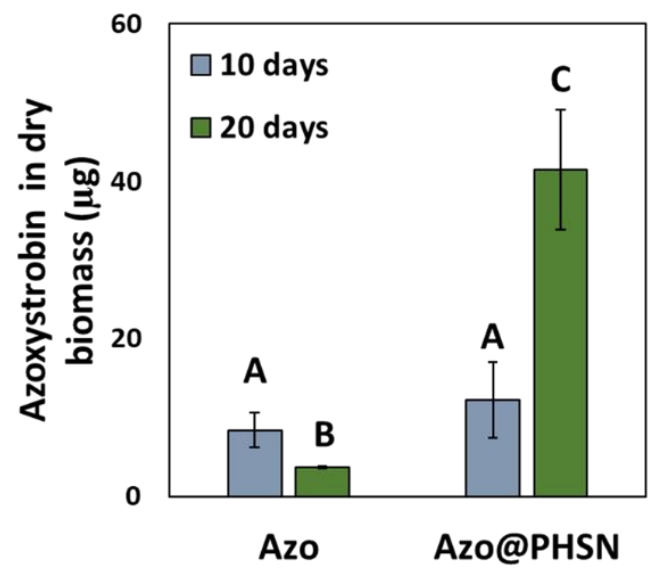

(C)

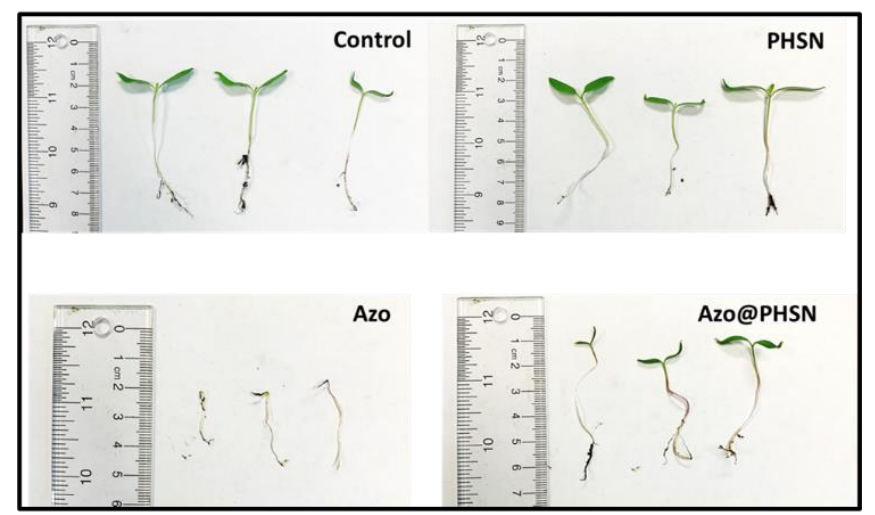

(D)

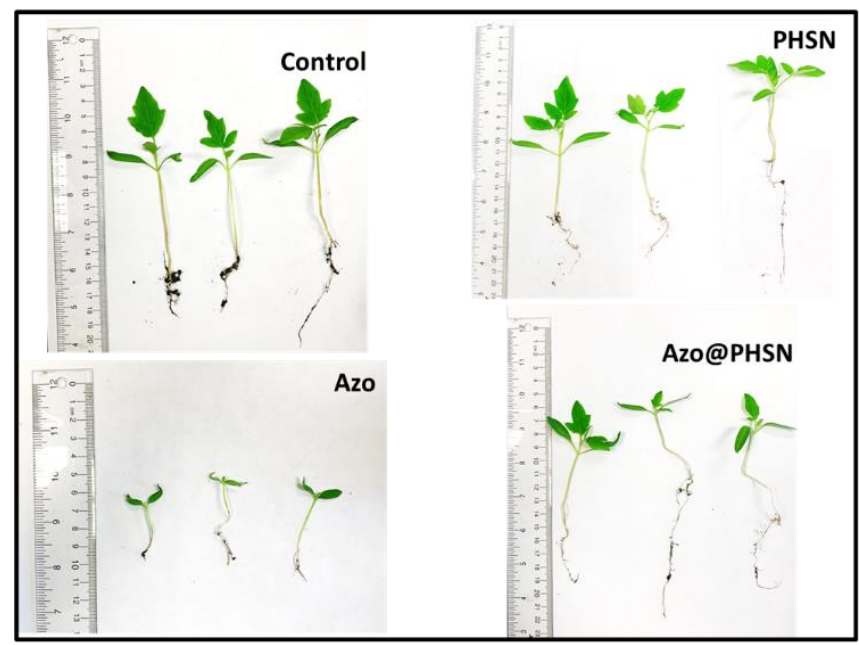

Figure 2. (A) Azoxystrobin concentration per unit of dry plant biomass at days 10 and 20 for Azo and Azo@PHSN treatments. The dry biomass was derived from plants after thorough rinse of the roots. (B) Azoxystrobin absolute uptake at days 10 and 20. Different letters $(A, B, C$ and $D)$ indicate significant statistical differences among samples (ANOVA one-way test, $\mathrm{p}<0.05$ threshold). (B) Photos of the plants harvested at day 10. (C) Photos of the plants harvested at day 20. 

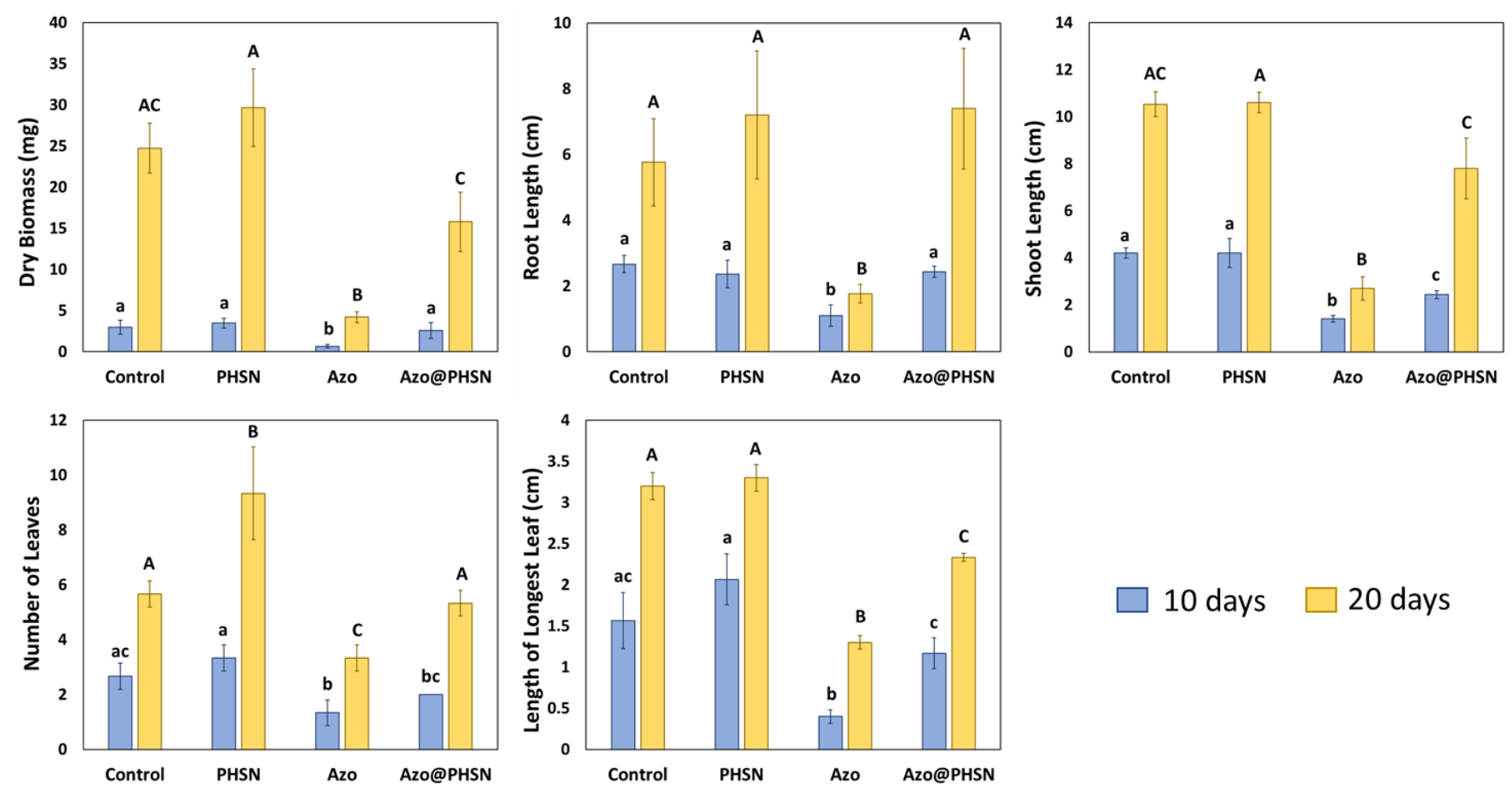

10 days $\square 20$ days

Figure 3. The measures of five phenotypic traits (dry mass, root length, shoot length, number of leaves and length of the longest leaf) as indicators of the impacts of each treatment in the plant growth. Different letters ( $a, b$ and $c$ for samples at day 10, $A, B$ and $C$ for samples at day 20) indicate significant statistical differences among samples (ANOVA one-way test, $\mathrm{p}<0.05$ threshold). 


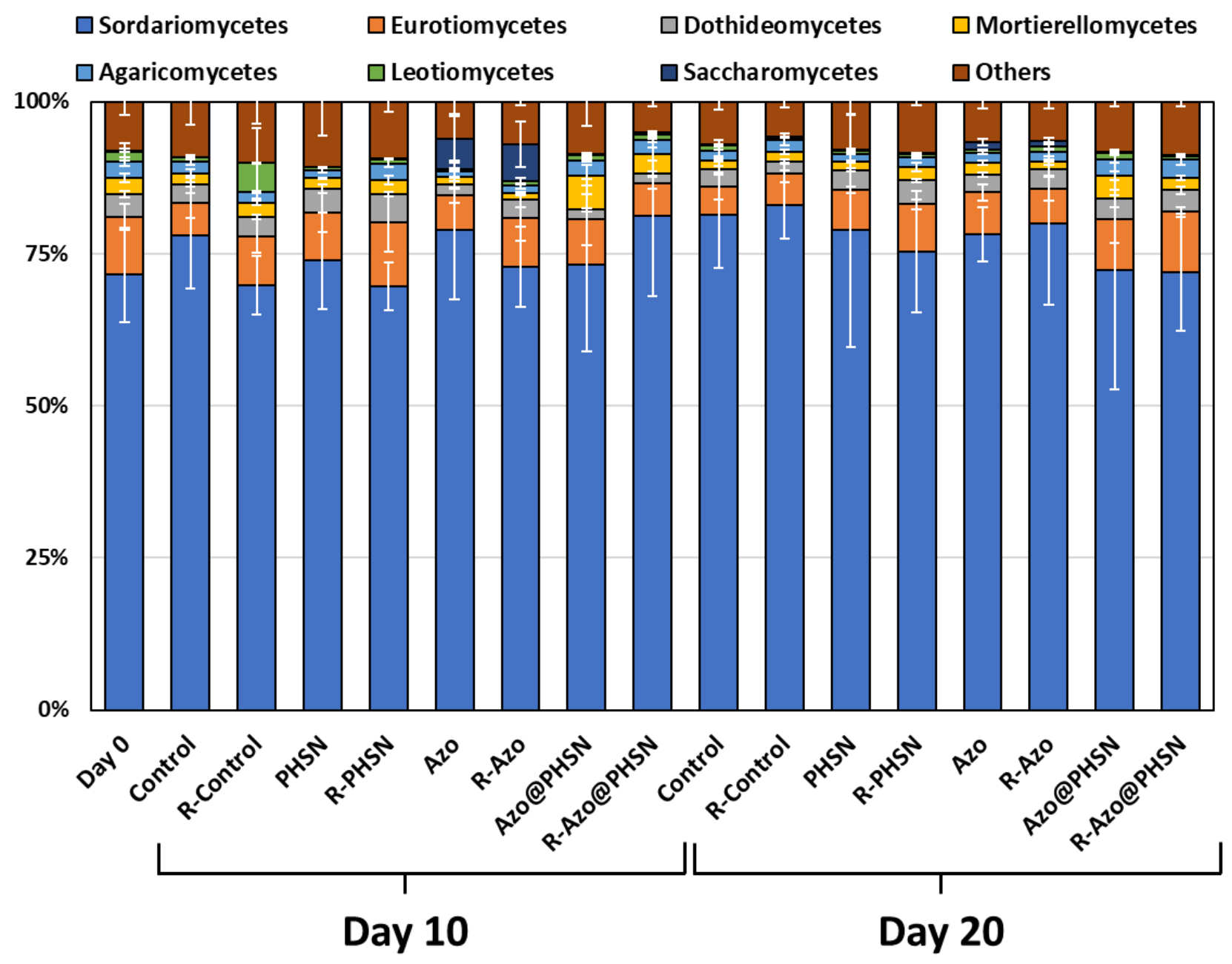

Figure 4. Relative taxonomic abundance of fungi communities at a class level. The communities obtained from loosely attached soil to the roots have the prefix $R$ - in front of the treatment ID. The communities obtained from bulk soil have no prefix in front of the treatment ID. 


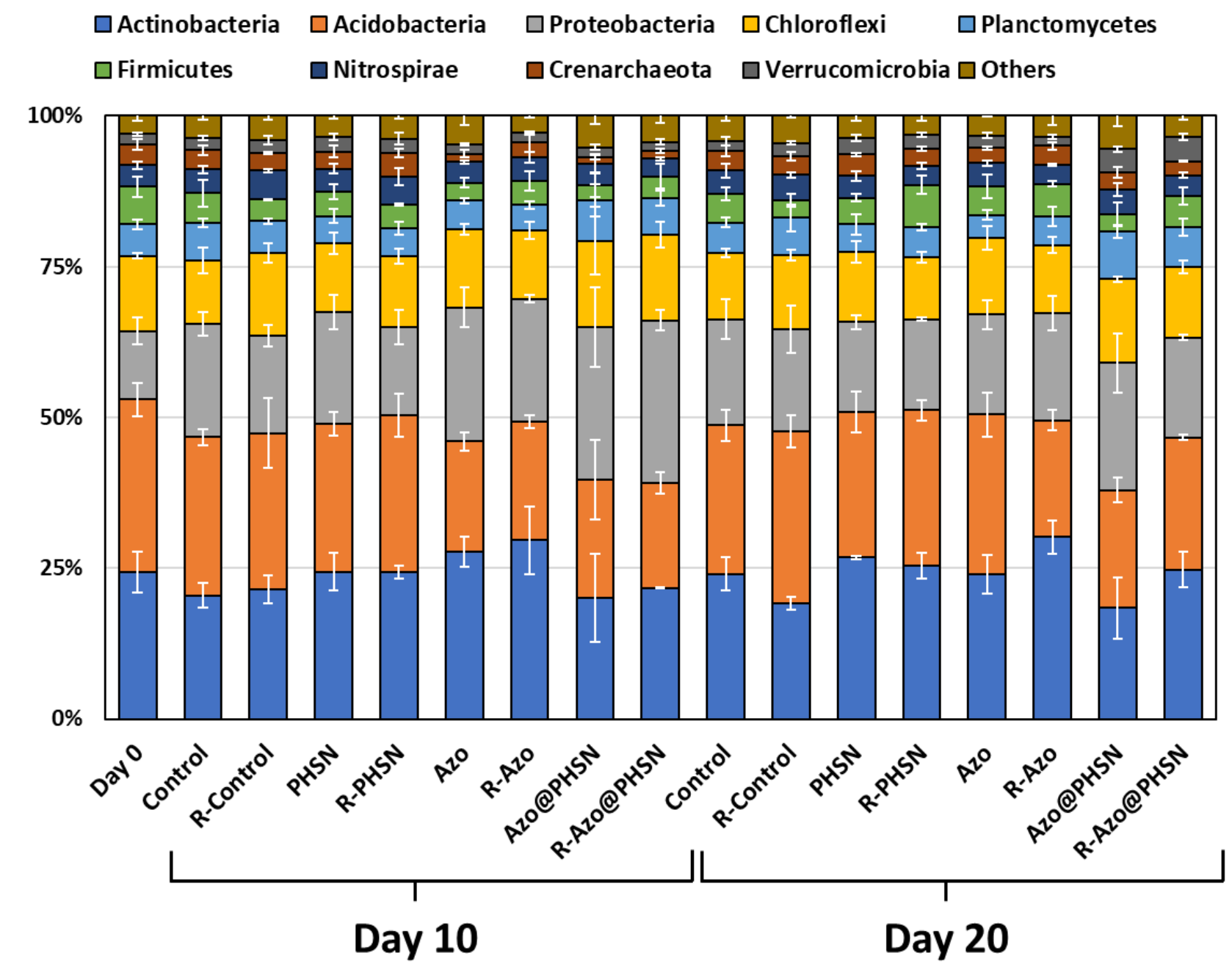

Figure 5. Relative taxonomic abundance of bacterial and archaea communities at a phylum level. The communities obtained from loosely attached soil to the roots have the prefix $R$ - in front of the treatment ID. The communities obtained from bulk soil have no prefix in front of the treatment ID. 


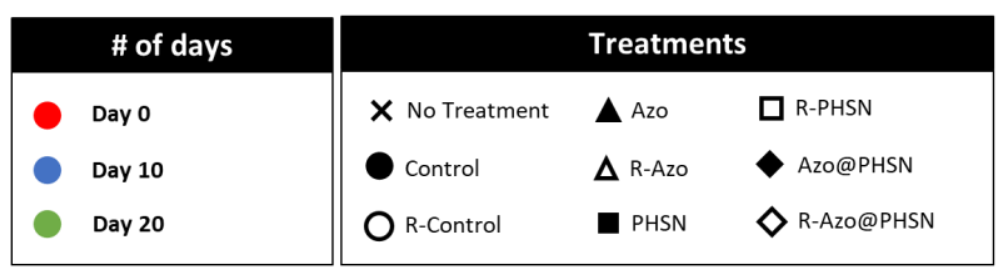
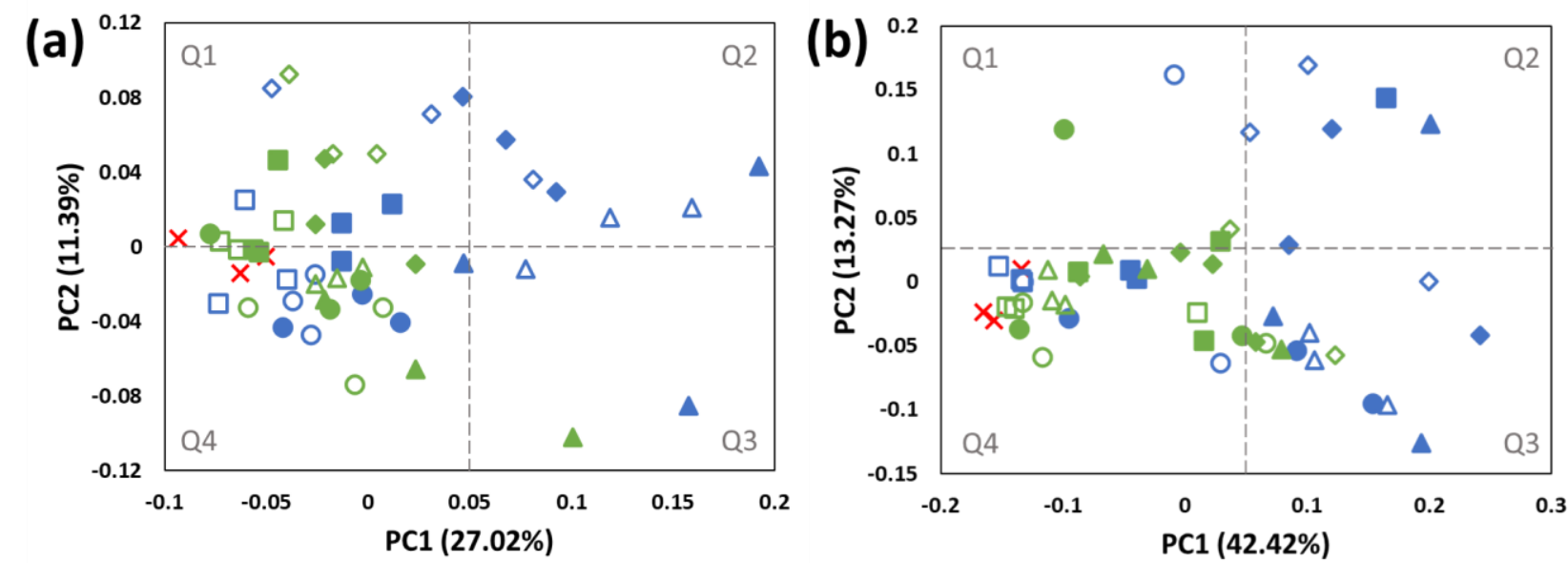

Figure 6. PCoAs for (a) bacterial and archaea communities and (b) fungi community. The communities obtained from loosely attached soil to the roots have the prefix $R$ - in front of the treatment ID. The communities obtained from bulk soil have no prefix in front of the treatment ID. 


\section{References}

1. M. Kah, R. S. Kookana, A. Gogos and T. D. Bucheli, A critical evaluation of nanopesticides and nanofertilizers against their conventional analogues, Nature Nanotechnology, 2018, 13, 677-684.

2. M. Kah, N. Tufenkji and J. C. White, Nano-enabled strategies to enhance crop nutrition and protection, Nature Nanotechnology, 2019, 14, 532-540.

3. G. V. Lowry, A. Avellan and L. M. Gilbertson, Opportunities and challenges for nanotechnology in the agri-tech revolution, Nature Nanotechnology, 2019, 14, 517-522.

4. T. Hofmann, G. V. Lowry, S. Ghoshal, N. Tufenkji, D. Brambilla, J. R. Dutcher, L. M. Gilbertson, J. P. Giraldo, J. M. Kinsella and M. P. Landry, Technology readiness and overcoming barriers to sustainably implement nanotechnology-enabled plant agriculture, Nature Food, 2020, 1, 416-425.

5. S. M. Rodrigues, P. Demokritou, N. Dokoozlian, C. O. Hendren, B. Karn, M. S. Mauter, O. A. Sadik, M. Safarpour, J. M. Unrine and J. Viers, Nanotechnology for sustainable food production: promising opportunities and scientific challenges, Environmental Science: Nano, 2017, 4, 767-781.

6. J. K. Patra, G. Das, L. F. Fraceto, E. V. R. Campos, M. del Pilar Rodriguez-Torres, L. S. Acosta-Torres, L. A. Diaz-Torres, R. Grillo, M. K. Swamy and S. Sharma, Nano based drug delivery systems: recent developments and future prospects, Journal of Nanobiotechnology, 2018, 16, 1-33.

7. W. H. Elmer and J. C. White, The use of metallic oxide nanoparticles to enhance growth of tomatoes and eggplants in disease infested soil or soilless medium, Environmental Science: Nano, 2016, 3, 1072-1079.

8. A. E. Pereira, R. Grillo, N. F. Mello, A. H. Rosa and L. F. Fraceto, Application of poly (epsilon-caprolactone) nanoparticles containing atrazine herbicide as an alternative technique to control weeds and reduce damage to the environment, Journal of Hazardous Materials, 2014, 268, 207-215. 
9. J. Tarafdar, R. Raliya, H. Mahawar and I. Rathore, Development of zinc nanofertilizer to enhance crop production in pearl millet (Pennisetum americanum), Agricultural Research, 2014, 3, 257-262.

10. R. Raliya and J. C. Tarafdar, ZnO nanoparticle biosynthesis and its effect on phosphorousmobilizing enzyme secretion and gum contents in Clusterbean (Cyamopsis tetragonoloba L.), Agricultural Research, 2013, 2, 48-57.

11. T. Prasad, P. Sudhakar, Y. Sreenivasulu, P. Latha, V. Munaswamy, K. R. Reddy, T. Sreeprasad, P. Sajanlal and T. Pradeep, Effect of nanoscale zinc oxide particles on the germination, growth and yield of peanut, Journal of Plant Nutrition, 2012, 35, 905-927.

12. D. Alidoust and A. Isoda, Effect of $\gamma \mathrm{Fe} 2 \mathrm{O} 3$ nanoparticles on photosynthetic characteristic of soybean (Glycine max (L.) Merr.): foliar spray versus soil amendment, Acta Physiologiae Plantarum, 2013, 35, 3365-3375.

13. M. Linglan, L. Chao, Q. Chunxiang, Y. Sitao, L. Jie, G. Fengqing and H. Fashui, Rubisco activase mRNA expression in spinach: modulation by nanoanatase treatment, Biological Trace Element Research, 2008, 122, 168-178.

14. A. Servin, W. Elmer, A. Mukherjee, R. De la Torre-Roche, H. Hamdi, J. C. White, P. Bindraban and C. Dimkpa, A review of the use of engineered nanomaterials to suppress plant disease and enhance crop yield, Journal of Nanoparticle Research, 2015, 17, 1-21.

15. M. El-Shetehy, A. Moradi, M. Maceroni, D. Reinhardt, A. Petri-Fink, B. RothenRutishauser, F. Mauch and F. Schwab, Silica nanoparticles enhance disease resistance in Arabidopsis plants, Nature Nanotechnology, 2020, 1-10.

16. M. Luyckx, J.-F. Hausman, S. Lutts and G. Guerriero, Silicon and plants: current knowledge and technological perspectives, Frontiers in Plant Science, 2017, 8, 411.

17. M. E. Abdel-Haliem, H. S. Hegazy, N. S. Hassan and D. M. Naguib, Effect of silica ions and nano silica on rice plants under salinity stress, Ecological Engineering, 2017, 99, 282-289.

18. M. Azeem, N. Iqbal, S. Kausar, M. T. Javed, M. S. Akram and M. A. Sajid, Efficacy of silicon priming and fertigation to modulate seedling's vigor and ion homeostasis of wheat 
(Triticum aestivum L.) under saline environment, Environmental Science and Pollution Research, 2015, 22, 14367-14371.

19. D. Coskun, D. T. Britto, W. Q. Huynh and H. J. Kronzucker, The role of silicon in higher plants under salinity and drought stress, Frontiers in Plant Science, 2016, 7, 1072.

20. D. A. Schurt, M. F. Cruz, K. J. Nascimento, M. C. Filippi and F. A. Rodrigues, Silicon potentiates the activities of defense enzymes in the leaf sheaths of rice plants infected by Rhizoctonia solani, Tropical Plant Pathology, 2014, 39, 457-463.

21. D. Ning, A. Song, F. Fan, Z. Li and Y. Liang, Effects of slag-based silicon fertilizer on rice growth and brown-spot resistance, Plos One, 2014, 9, e102681.

22. D. Sun, H. I. Hussain, Z. Yi, J. E. Rookes, L. Kong and D. M. Cahill, Mesoporous silica nanoparticles enhance seedling growth and photosynthesis in wheat and lupin, Chemosphere, 2016, 152, 81-91.

23. D. K. Tripathi, S. Singh, V. P. Singh, S. M. Prasad, N. K. Dubey and D. K. Chauhan, Silicon nanoparticles more effectively alleviated UV-B stress than silicon in wheat (Triticum aestivum) seedlings, Plant Physiology and Biochemistry, 2017, 110, 70-81.

24. D. K. Tripathi, V. P. Singh, S. M. Prasad, D. K. Chauhan and N. K. Dubey, Silicon nanoparticles (SiNp) alleviate chromium (VI) phytotoxicity in Pisum sativum (L.) seedlings, Plant Physiology and Biochemistry, 2015, 96, 189-198.

25. J. Cui, Y. Li, Q. Jin and F. Li, Silica nanoparticles inhibit arsenic uptake into rice suspension cells via improving pectin synthesis and the mechanical force of the cell wall, Environmental Science: Nano, 2020, 7, 162-171.

26. M. H. Siddiqui and M. H. Al-Whaibi, Role of nano-SiO2 in germination of tomato (Lycopersicum esculentum seeds Mill.), Saudi Journal of Biological Sciences, 2014, 21, 13-17.

27. L. Bao-Shan, L. Chun-hui, F. Li-jun, Q. Shu-chun and Y. Min, Effect of TMS (nanostructured silicon dioxide) on growth of Changbai larch seedlings, Journal of Forestry Research, 2004, 15, 138-140. 
28. L. Tian, J. Shen, G. Sun, B. Wang, R. Ji and L. Zhao, Foliar Application of SiO2 Nanoparticles Alters Soil Metabolite Profiles and Microbial Community Composition in the Pakchoi (Brassica chinensis L.) Rhizosphere Grown in Contaminated Mine Soil, Environmental Science \& Technology, 2020, 54, 13137-13146.

29. T. M. Abdelrahman, X. Qin, D. Li, I. A. Senosy, M. Mmby, H. Wan, J. Li and S. He, Pectinase-responsive carriers based on mesoporous silica nanoparticles for improving the translocation and fungicidal activity of prochloraz in rice plants, Chemical Engineering Journal, 2021, 404, 126440.

30. O. Plohl, S. Gyergyek and L. F. Zemljič, Mesoporous silica nanoparticles modified with $\mathrm{N}$-rich polymer as a potentially environmentally-friendly delivery system for pesticides, Microporous and Mesoporous Materials, 2021, 310, 110663.

31. J. Feng, W. Chen, Y. Shen, Q. Chen, J. Yang, M. Zhang, W. Yang and S. Yuan, Fabrication of abamectin-loaded mesoporous silica nanoparticles by emulsion-solvent evaporation to improve photolysis stability and extend insecticidal activity, Nanotechnology, 2020, 31, 345705.

32. F. Liu, L.-X. Wen, Z.-Z. Li, W. Yu, H.-Y. Sun and J.-F. Chen, Porous hollow silica nanoparticles as controlled delivery system for water-soluble pesticide, Materials Research Bulletin, 2006, 41, 2268-2275.

33. Z.-Z. Li, S.-A. Xu, L.-X. Wen, F. Liu, A.-Q. Liu, Q. Wang, H.-Y. Sun, W. Yu and J.-F. Chen, Controlled release of avermectin from porous hollow silica nanoparticles: Influence of shell thickness on loading efficiency, UV-shielding property and release, Journal of Controlled Release, 2006, 111, 81-88.

34. L. X. Wen, Z. Z. Li, H. K. Zou, A. Q. Liu and J. F. Chen, Controlled release of avermectin from porous hollow silica nanoparticles, Pest Management Science, 2005, 61, 583-590.

35. Y. Xu, C. Xu, Q. Huang, L. Cao, F. Teng, P. Zhao and M. Jia, Size Effect of Mesoporous Silica Nanoparticles on Pesticide Loading, Release, and Delivery in Cucumber Plants, Applied Sciences, 2021, 11, 575. 
36. V. Bueno and S. Ghoshal, Self-Assembled Surfactant-Templated Synthesis of Porous Hollow Silica Nanoparticles: Mechanism of Formation and Feasibility of Post-Synthesis Nanoencapsulation, Langmuir, 2020, 36, 14633-14643.

37. D. W. Bartlett, J. M. Clough, J. R. Godwin, A. A. Hall, M. Hamer and B. Parr Dobrzanski, The strobilurin fungicides, Pest Management Science, 2002, 58, 649-662.

38. J. Bazuin, Azoxystrobin found to be phytotoxic to certain varieties of apples, USEPA Pesticide Program Update, 1999, 21.

39. N. Lalancette, W. Cowgill, J. Compton and K. Foster, Proceedings of the 76th Cumberland Shenandoah Fruit Workers Conference, 2000.

40. A. Landers, Drift reduction in the vineyards of New York and Pennsylvania, Aspects of Applied Biology, 2000, 67-73.

41. L. Jin, C. Chen, J. Wang, Y. Chen and M.-g. Zhou, Activity of azoxystrobin and SHAM of four plant pathogens, Scientia Agricultura Sinica, 2007, 10.

42. V. Bueno, A. Bosi, T. Tosco and S. Ghoshal, Mobility of Solid and Porous Hollow SiO2 Nanoparticles in Saturated Porous Media: Impacts of Surface and Particle Structure, Journal of Colloid and Interface Science, 2022, 606, 480-490.

43. United States Environmental Protection Agency, Pesticide Fact Sheet: Azoxystrobin, https://www3.epa.gov/pesticides/chem_search/reg_actions/registration/fs_PC-128810_07-Feb97.pdf, (accessed 7 March 2021).

44. Health Canada, Proposed Maximum Residue Limit PMRL2020-48, Azoxystrobin, https://www.canada.ca/en/health-canada/services/consumer-product-safety/pesticides-pestmanagement/public/consultations/proposed-maximum-residue-limit/2020/azoxystrobinvarious/document.html, (accessed 29 September 2021).

45. S. Lehotay, AOAC official method 2007.01 pesticide residues in foods by acetonitrile extraction and partitioning with Magnesium Sulfate, Journal of AOAC International, 2007, 90, 485-520. 
46. M. Kah, A.-K. Weniger and T. Hofmann, Impacts of (Nano) formulations on the Fate of an Insecticide in Soil and Consequences for Environmental Exposure Assessment, Environmental Science \& Technology, 2016, 50, 10960-10967.

47. V. L. Singer, L. J. Jones, S. T. Yue and R. P. Haugland, Characterization of PicoGreen reagent and development of a fluorescence-based solution assay for double-stranded DNA quantitation, Analytical Biochemistry, 1997, 249, 228-238.

48. S. J. Ahn, J. Costa and J. Rettig Emanuel, PicoGreen quantitation of DNA: effective evaluation of samples pre-or post-PCR, Nucleic Acids Research, 1996, 24, 2623-2625.

49. E. Bolyen, J. R. Rideout, M. R. Dillon, N. A. Bokulich, C. Abnet, G. A. Al-Ghalith, H. Alexander, E. J. Alm, M. Arumugam and F. Asnicar, QIIME 2: Reproducible, interactive, scalable, and extensible microbiome data science, Report 2167-9843, PeerJ Preprints, 2018.

50. B. J. Callahan, P. J. McMurdie, M. J. Rosen, A. W. Han, A. J. A. Johnson and S. P. Holmes, DADA2: high-resolution sample inference from Illumina amplicon data, Nature Methods, 2016, 13, 581-583.

51. Q. Wang, G. M. Garrity, J. M. Tiedje and J. R. Cole, Naive Bayesian classifier for rapid assignment of rRNA sequences into the new bacterial taxonomy, Applied and Environmental Microbiology, 2007, 73, 5261-5267.

52. D. McDonald, M. N. Price, J. Goodrich, E. P. Nawrocki, T. Z. DeSantis, A. Probst, G. L. Andersen, R. Knight and P. Hugenholtz, An improved Greengenes taxonomy with explicit ranks for ecological and evolutionary analyses of bacteria and archaea, The ISME Journal, 2012, 6, 610618.

53. U. Kõljalg, R. H. Nilsson, K. Abarenkov, L. Tedersoo, A. F. Taylor, M. Bahram, S. T. Bates, T. D. Bruns, J. Bengtsson - Palme and T. M. Callaghan, Towards a unified paradigm for sequence - based identification of fungi. Molecular Ecology, 2013, 22, 5271-5277.

54. D. L. Taylor, W. A. Walters, N. J. Lennon, J. Bochicchio, A. Krohn, J. G. Caporaso and T. Pennanen, Accurate estimation of fungal diversity and abundance through improved lineagespecific primers optimized for Illumina amplicon sequencing, Applied and Environmental Microbiology, 2016, 82, 7217-7226. 
55. D. P. Faith, Conservation evaluation and phylogenetic diversity, Biological Conservation, 1992, 61, 1-10.

56. E. C. Pielou, The measurement of diversity in different types of biological collections, Journal of Theoretical Biology, 1966, 13, 131-144.

57. C. A. Lozupone and R. Knight, Global patterns in bacterial diversity, Proceedings of the National Academy of Sciences, 2007, 104, 11436-11440.

58. T. Lueders, M. Manefield and M. W. Friedrich, Enhanced sensitivity of DNA- and rRNAbased stable isotope probing by fractionation and quantitative analysis of isopycnic centrifugation gradients, Environmental Microbiology, 2004, 6, 73-78.

59. T. Lueders, B. Wagner, P. Claus and M. W. Friedrich, Stable isotope probing of rRNA and DNA reveals a dynamic methylotroph community and trophic interactions with fungi and protozoa in oxic rice field soil, Environmental Microbiology, 2004, 6, 60-72.

60. C. Edwards, Factors that affect the persistence of pesticides in plants and soils, Pesticide Chemistry-3, 1975, 39-56.

61. V. Bansal, A. Ahmad and M. Sastry, Fungus-mediated biotransformation of amorphous silica in rice husk to nanocrystalline silica, Journal of the American Chemical Society, 2006, 128, 14059-14066.

62. J. H. LANGE, A study of apple trees exposed to the fungicide azoxystrobin and mixture interactions with surfactants, International Journal of Environmental Studies, 2004, 61, 173-187.

63. J. Godwin, V. Anthony, J. Clough and C. R. Godfrey, ICIA5504: A novel, broad spectrum, systemic beta-methoxyacrylate fungicide, 1992.

64. M. Baćmaga, J. Wyszkowska and J. Kucharski, Bioaugmentation of soil contaminated with azoxystrobin, Water, Air, \& Soil Pollution, 2017, 228, 1-9.

65. X. Wang, Z. Lu, H. Miller, J. Liu, Z. Hou, S. Liang, X. Zhao, H. Zhang and T. Borch, Fungicide azoxystrobin induced changes on the soil microbiome, Applied Soil Ecology, 2020, 145, 103343. 
66. M. Baćmaga, J. Kucharski and J. Wyszkowska, Microbial and enzymatic activity of soil contaminated with azoxystrobin, Environmental Monitoring and Assessment, 2015, 187, 1-15.

67. E. Adetutu, A. Ball and A. Osborn, Azoxystrobin and soil interactions: degradation and impact on soil bacterial and fungal communities, Journal of Applied Microbiology, 2008, 105, 1777-1790.

68. G. D. Bending, M. S. Rodríguez-Cruz and S. D. Lincoln, Fungicide impacts on microbial communities in soils with contrasting management histories, Chemosphere, 2007, 69, 82-88.

69. F. Wang, X. Li, L. Zhu, Z. Du, C. Zhang, J. Wang, J. Wang and D. Lv, Responses of Soil Microorganisms and Enzymatic Activities to Azoxystrobin in Cambisol, Polish Journal of Environmental Studies, 2018, 27.

70. A. C. Kennedy and T. L. Stubbs, Soil microbial communities as indicators of soil health, 2006.

71. S. Albrecht, Y. Okon, J. Lonnquist and R. Burris, Nitrogen Fixation by Corn Azospirillum Associations in a Temperate Climate 1, Crop Science, 1981, 21, 301-306.

72. G. W. Bruehl, Soilborne Plant Pathogens, Macmillan publishing company, 1987.

73. R. Schroll, H. H. Becher, U. Dörfler, S. Gayler, S. Grundmann, H. P. Hartmann and J. Ruoss, Quantifying the effect of soil moisture on the aerobic microbial mineralization of selected pesticides in different soils, Environmental Science \& Technology, 2006, 40, 3305-3312.

74. T. Henriksen and T. Breland, Nitrogen availability effects on carbon mineralization, fungal and bacterial growth, and enzyme activities during decomposition of wheat straw in soil, Soil Biology and Biochemistry, 1999, 31, 1121-1134.

75. A. Botha, The importance and ecology of yeasts in soil, Soil Biology and Biochemistry, 2011, 43, 1-8.

76. A. M. Spain, L. R. Krumholz and M. S. Elshahed, Abundance, composition, diversity and novelty of soil Proteobacteria, The ISME Journal, 2009, 3, 992-1000. 
77. M. Mhete, P. N. Eze, T. O. Rahube and F. O. Akinyemi, Soil properties influence bacterial abundance and diversity under different land-use regimes in semi-arid environments, Scientific African, 2020, 7, e00246.

78. C.-C. Lo, Effect of pesticides on soil microbial community, Journal of Environmental Science and Health Part B, 2010, 45, 348-359. 